\title{
On Hybrid Adaptive and Pinning Consensus for Multiagent Networks
}

\author{
Lianghao Ji, Yi Tang, and Qun Liu \\ Chongqing Key Laboratory of Computational Intelligence, Chongqing University of Posts and Telecommunications, \\ Chongqing 400065, China
}

Correspondence should be addressed to Lianghao Ji; lianghao.ji@gmail.com

Received 24 May 2016; Revised 1 September 2016; Accepted 25 October 2016

Academic Editor: Jean J. Loiseau

Copyright (C) 2016 Lianghao Ji et al. This is an open access article distributed under the Creative Commons Attribution License, which permits unrestricted use, distribution, and reproduction in any medium, provided the original work is properly cited.

The consensus problems for both continuous-time and discrete-time multiagent networks are deeply investigated by adopting hybrid adaptive and pinning control laws, respectively. Particularly, the topology of the networks needs to neither be symmetric nor contain a directed spanning tree and some useful criteria are addressed analytically. Simultaneously, a comprehensive pinning scheme is proposed as well which shows that the nodes with zero in-degree need to be pinned primarily in order to guarantee the system to achieve consensus, and then the nodes whose out-degrees are bigger than their in-degrees can give priority to be pinned compared to other nodes for improving the convergence rate of the system, whereas it is also interesting to find out that the regular rule does not always hold, that is, the more nodes are selected to be pinned, the faster the system will converge. Finally, the validity of our theoretical findings is verified by several numerical examples.

\section{Introduction}

The consensus problems aim to design some control strategies and protocols such that the whole network can achieve stability, which are the fundamental problem in the field of distributed cooperative control of complex networks. Meanwhile, they also have attracted increasing attention in various research fields because of their wide applications. So far, many related research works have been successively reported, which can be seen in [1-10] and the references therein.

In fact, dynamic network cannot achieve the consensus asymptotically by itself in the normal case. Therefore, control strategy and controllers should be designed and applied to the nodes of the network. But it is impractical to control all nodes in a network and costly as well. So pinning control can provide a feasible method to improve these problems. Up to now, there are a lot of excellent research works that have been reported for pinning consensus and pinning synchronization. Like in [11], the results showed that the system with undirected topology or containing a directed spanning tree can achieve synchronization by pinning a single node when the coupling strength between the agents was large enough. In $[12,13]$, the problems about the pinning consensus of networks with undirected topology were studied and some criteria are proposed as well. Subsequently, Chen et al. [14] investigated the pinning consensus of multiagent networks with strongly connected topology. In [15], the sufficient condition was proposed which can guarantee the directed network with reducible coupling matrix to realize the synchronization. In [16], the pinning global synchronization of both directed and undirected complex networks was discussed and some criteria were presented analytically. The impulsive synchronization of the networks with undirected or strongly connected topology was studied in [17] by adopting pinning stratagem. In [18], the controllability of pinning for the complex networks whose topology contains a directed spanning tree was presented. For second-order discrete-time multiagent networks with switching topology, pinning consensus problem is discussed in [19]. The results showed that consensus could be reached if the union digraph of the associated topology had a directed spanning tree. In [20], pinning consensus of multiagent networks via a single impulsive controller was investigated. The topology of the system is strongly connected and with a spanning tree. In [21], by using M-matrix, the leader-following consensus 
problem of multiagent systems was considered. Meanwhile, the authors also pointed out that the system can be easier to achieve consensus when more agents were pinned or pinning gains were increased. Via adapting pinning control, leaderfollowing consensus of second-order multiagent systems was investigated in [22]. Meanwhile, the problems about what kind of nodes and how many nodes should be pinned were also been addressed. The results showed that the agents who cannot be influenced by any other agent must be pinned. In addition, according the degree-differences of the agent, the authors also gave an adaptive pinning scheme to select the pinning agents. The consensus of multiagent networks with transmission and pinning delays was investigated and the related criteria were addressed as well when assuming that the topology is strongly connected and at least one node was pinned in [23]. In [24], leader-following consensus problem of multiagent networks with delays is discussed by using adaptive pinning intermittent control method. By dividing the diagraph into multiple components, the results indicated that the root node of each component is needed to be pinned. In addition, the nodes satisfying the proposed limitation condition can also be selected according to out-degree and in-degree of the nodes. In [25], $\mathrm{Hu}$ et al. investigated the second-order consensus problems of heterogeneous multiagent system via adaptive and pinning methods. In addition, some research works about group consensus and cluster synchronization by introducing pinning strategy have also been published, as seen in [26-32] and the references therein.

From the relevant research works mentioned above, it is not difficult to find out there exist some more conservative assumptions in [11-20, 23, 26, 27, 30]. And these assumptions imply that the network owns a special topology, such as an undirected graph, strongly connected or containing a directed spanning tree, and so forth. In addition, as we know, it is very difficult to compute and verify the control gains of the system with the expansion of the scale of the network, so adaptive control law naturally becomes a good choice in practical application.

Inspired by the related research works, we will investigate the consensus problems for both continuous-time and discrete-time dynamic multiagent networks by adopting adaptive and pinning control laws, respectively. The main contribution of this paper lies in the following aspects. First, some useful criteria are proposed analytically, which can guarantee the multiagent network to achieve consensus. The topology of the complex system needs to neither be symmetrical nor contain a directed spanning tree. Namely, we do not limit the topology as an undirected graph or strongly connected graph or with a reducible coupling matrix. Therefore, the results we addressed are more general and practical. Second, there is an effective and comprehensive pinning scheme that has been derived for these two kinds of system, respectively. How to choose and how many nodes should be selected are two fundamental problems with pinning control. Considering the convergence performance and convergence rate of the system, we give a reference answer to these two problems. The validity has been verified by the simulation examples. Third, in order to obtain the appropriate value of control gains, adaptive control laws have been designed and adopted which is useful when the scale of a network expands unceasingly.

The rest of this paper is organized as follows. In Section 2, some preliminaries are briefly outlined. The main results of hybrid adaptive and pinning consensus for both continuoustime and discrete-time multiagent networks are addressed in Section 3. In Section 4, some examples are illustrated to verify our theoretical results and conclusions are drawn in Section 5.

Notation. In this paper, let $\mathbb{R}, \mathbb{N}$, and $\mathbb{C}$ denote the sets of real, natural, and complex numbers, respectively. For $z \in \mathbb{C}, \operatorname{Re}(z)$ and $\operatorname{Im}(z)$ represent its real and imaginary parts, respectively. Let $I_{n}$ denote an identity matrix with $n$-dimensional. For a real matrix $A \in \mathbb{R}^{n \times n}$, let $A^{T}$ and $A_{s}=\left(A+A^{T}\right) / 2$ be its transpose and symmetric part, respectively. And for a real square matrix $A$, denote its inverse and eigenvalues by $A^{-1}$ and $\lambda_{i}(A)$, and denote $A>0(A<0)$, if $A$ is positive (negative) definite, respectively. $\otimes$ denotes the Kronecker product.

\section{Preliminaries and Problem Statement}

In this section, some preliminaries and useful lemmas will be listed.

2.1. Graph Theory. In a multiagent network, we can represent each agent and the information exchange among the agents as a node and an edge of a weighted directed graph, respectively. For convenience, let $g=(V, E, A)$ denote a weighted directed graph, where $V=\left\{v_{1}, v_{2}, \ldots, v_{N}\right\}$ is the node set, $\mathbb{N}=$ $\{1,2, \ldots, N\}$ denotes the node index set, $E \subseteq V \times V$ is the edge set, and $A=\left(a_{i j}\right) \in \mathbb{R}^{N \times N}$ is the weighted adjacency matrix. If the edge $e_{i j} \in E, a_{i j}>0$, which means node $v_{i}$ can receive information from node $v_{j}$. Otherwise, $a_{i j}=0$. In this paper, we assumed that $a_{i i}=0$ for all $i \in \mathbb{N}$. Meanwhile, the Laplacian matrix associated with the matrix $G$ is defined as

$$
L=\left(l_{i j}\right)_{N \times N}= \begin{cases}-a_{i j}, & i \neq j \\ \sum_{j=1, j \neq i}^{N} a_{i j}, & i=j .\end{cases}
$$

2.2. Problem Statement. Consider a network $g$ consisting of $N$ linearly coupled identical nodes which owns the following dynamics outlined as

$$
\dot{x}_{i}(t)=c \sum_{j=1}^{N} G_{i j} \Gamma\left(x_{j}(t)-x_{i}(t)\right), \quad i \in \mathbb{N},
$$

where $x_{i}(t)=\left[x_{i 1}, x_{i 2}, \ldots, x_{i n}\right]^{T} \in \mathbb{R}^{n}$ is the state vector of the node $v_{i}$ at time $t$; $c$ is the coupling strength of the system and $c>0$; matrix $\Gamma>0 \in \mathbb{R}^{n \times n}$ describes the inner coupling between the agents; and $G=\left(G_{i j}\right) \in \mathbb{R}^{N \times N}$ is the weighted adjacency matrix.

Definition 1. For any initial state values $x_{i}(0)$, network (2) can be said to achieve consensus asymptotically if it follows that $\lim _{t \rightarrow+\infty}\left\|x_{i}(t)-x_{j}(t)\right\|=0, \forall i, j \in \mathbb{N}$. 
Definition 2 (see [33]). For a nonsingular square matrix $A=$ $\left(a_{i j}\right) \in \mathbb{R}^{N \times N}$, if $a_{i j}<0, i \neq j$, and all the elements of matrix $A^{-1}$ are nonnegative, matrix $A$ can be called an M-matrix.

Lemma 3 (see [33]). For a nonsingular square matrix $A=$ $\left(a_{i j}\right) \in \mathbb{R}^{N \times N}$, the following three statements are equivalent when $a_{i j} \leq 0, i \neq j$ holds:

(i) Matrix $A$ is an M-matrix.

(ii) $\operatorname{Re}\left(\lambda_{i}(A)\right)>0, i \in \mathbb{N}$.

(iii) $(\Xi A)_{s}>0$ holds, where $\Xi=\left\{\varsigma_{1}, \varsigma_{2}, \ldots, \varsigma_{N}\right\}$ is a positive definite diagonal matrix.

Lemma 4 (see [33]). For matrices A, B, C, and D with suitable dimensions, the following two equations can be satisfied:

(i) $(A+B) \otimes C=A \otimes C+B \otimes C$.

(ii) $(A \otimes B)(C \otimes D)=(A C) \otimes(B D)$.

\section{Main Results}

In this section, we will discuss the hybrid adaptive and pinning consensus problems for both continuous-time and discrete-time multiagent networks, respectively.

3.1. Adaptive Pinning Consensus for Continuous-Time Multiagent Network. To make system (2) achieve the consensus asymptotically, some pinning controllers will be introduced to parts of its nodes. Therefore, network (2) can be described as follows:

$$
\begin{aligned}
& \dot{x}_{i}(t)=c \sum_{j=1}^{N} G_{i j} \Gamma\left(x_{j}(t)-x_{i}(t)\right)+u_{i}(t), \quad i \in \mathbb{N}, \\
& u_{i}(t)=-c d_{i} \Gamma\left(x_{i}(t)-x^{*}\right), \quad i \in \mathbb{N},
\end{aligned}
$$

where $u_{i}(t) \in \mathbb{R}^{n}$ are linear feedback controllers and the control gains $d_{i}$ are defined as follows: if node $v_{i}$ is pinned, $d_{i}>0$; otherwise, $d_{i}=0 . x^{*} \in \mathbb{R}^{n}$ is the desired equilibrium state value.

Define the error system as

$$
e_{i}(t)=x_{i}(t)-x^{*}, \quad i \in \mathbb{N} \text {. }
$$

By Definition 1, one can know that pinning consensus aims to design controllers for network (3) to achieve $\lim _{t \rightarrow+\infty}\left\|e_{i}(t)\right\|=0, i \in \mathbb{N}$.

From (3) and (4), it follows that

$$
\dot{e}_{i}(t)=c \sum_{j=1}^{N} G_{i j} \Gamma\left(e_{j}(t)-e_{i}(t)\right)-c d_{i} \Gamma e_{i}(t), \quad i \in \mathbb{N} .
$$

Based on the definition of Laplacian matrix, (6) can be transformed as

$$
\dot{e}_{i}(t)=-c \sum_{j=1}^{N} l_{i j} \Gamma e_{j}(t)-c d_{i} \Gamma e_{i}(t), \quad i \in \mathbb{N} .
$$

Let $e(t)=\left[e^{T}{ }_{1}(t), e^{T}{ }_{2}(t), \ldots, e^{T}{ }_{N}(t)\right]^{T}$; by Lemma $4,(7)$ can be rewritten as

$$
\dot{e}(t)=-c(L+D) \otimes \Gamma e(t) .
$$

To reduce the cost of control, we usually hope to choose suitable pinning control gains and get its values as small as possible. Next, we will introduce the adaptive control strategy to solve this problem.

Considering the following protocol of adaptive controlled gains,

$$
\dot{d}_{i}=\left(x_{i}(t)-x^{*}\right)^{T} \Gamma\left(x_{i}(t)-x^{*}\right), \quad i \in \mathbb{N},
$$

in view of (5), rewrite (9) as

$$
\dot{d}_{i}=e_{i}(t)^{T} \Gamma e_{i}(t), \quad i \in \mathbb{N} .
$$

Theorem 5. Suppose that network (3) contains $N$ nodes and $\Gamma$ is a positive definite diagonal matrix; pinning consensus of system (3) with general connected topology can be achieved asymptotically when $-c(L+\widehat{D})_{s}<0$ holds, where matrix $L$ is the Laplacian matrix associated with the adjacency matrix of network (3), $\widehat{D}=\operatorname{diag}\left\{\widehat{d}_{1}, \widehat{d}_{2}, \ldots, \widehat{d}_{N}\right\}$, and $\widehat{d}_{i}>0$ if node $v_{i}$ is selected to be pinned and its value can be given by (9); else $\widehat{d}_{i}=0$.

Proof. Define a Lyapunov functional candidate for network (3) as

$$
V(t)=\frac{1}{2} e_{i}^{T}(t) e_{i}(t)+\frac{c}{2}\left(d_{i}-\widehat{d}_{i}\right)^{2}, \quad i \in \mathbb{N}
$$

Calculating the time derivative of $V(t)$ along the trajectories of (8), one can have

$$
\dot{V}(t)=e_{i}^{T}(t) \dot{e}_{i}(t)+c\left(d_{i}-\widehat{d}_{i}\right) \dot{d}_{i}, \quad i \in \mathbb{N},
$$

and in view of (8) and (10), we can get

$$
\begin{aligned}
\dot{V}(t)= & e_{i}^{T}(t) \dot{e}_{i}(t)+c\left(d_{i}-\widehat{d}_{i}\right) \dot{d}_{i} \\
= & e_{i}^{T}(t)[-c(L+D) \otimes \Gamma] e_{i}(t) \\
& +c\left(d_{i}-\widehat{d}_{i}\right) e_{i}^{T}(t) \Gamma e_{i}(t), \quad i \in \mathbb{N} .
\end{aligned}
$$

By Lemma 4, rewrite (13) in the matrix form as

$$
\begin{aligned}
\dot{V}(t) & =e^{T}(t)[-c(L+\widehat{D}) \otimes \Gamma] e(t) \\
& =e^{T}(t)\left[-c(L+\widehat{D})_{s} \otimes \Gamma\right] e(t) .
\end{aligned}
$$

As $\Gamma>0$, if $-c(L+\widehat{D})_{s}<0$ holds, it follows that $\dot{V}(t)<0$. Based on the theory of Lyapunov, the equation $\lim _{t \rightarrow+\infty}\left\|e_{i}(t)\right\|=0, i \in \mathbb{N}$, can be satisfied. Thus, network (3) can realize pinning consensus asymptotically. The proof is completed.

Remark 6. The results in Theorem 5 give a common criterion to guarantee network (3) with general connected topology to 
realize pinning consensus asymptotically, whereas the computational burden is becoming more and more challenging and it is also difficult to select the pinned nodes with the expanding of network scale. Next, we will continue to deeply discuss these problems.

Theorem 7. Suppose that network (3) contains $N$ nodes and $\Gamma$ is a positive definite diagonal matrix; the desired pinning consensus of network (3) with general connected topology can be achieved asymptotically if the matrix $L+\widehat{D}$ is an M-matrix, where matrices $L$ and $\widehat{D}$ are the same as those in Theorem 5 .

Proof. If the matrix $L+\widehat{D}$ is an M-matrix, by Lemma 3, one can get $(L+\widehat{D})_{s}>0$. Since $\Gamma>0$ is a positive definite diagonal matrix, in view of (14), it follows that $\dot{V}(t)<0$. By the theory of Lyapunov, we know that the consensus of network (3) can be achieved. The proof is completed.

Remark 8. From Definition 2, one can find the results in Theorem 7 cannot be applied when $a_{i j}<0$. It will be one of the problems needed to be further researched in the future.

Corollary 9. Suppose that network (3) contains $N$ nodes and $\Gamma$ is a positive definite diagonal matrix; pinning consensus for network (3) with general connected topology can be achieved if $L+\widehat{D}$ is a nonsingular matrix and the following conditional inequality $\operatorname{Re}\left(\lambda_{i}(L+\widehat{D})\right)>0, i \in \mathbb{N}$, holds.

By Lemma 3, as the condition $\operatorname{Re}\left(\lambda_{i}(L+\widehat{D})\right)>0, i \in \mathbb{N}$ is equivalent with that $L+\widehat{D}$ is an M-matrix if $L+\widehat{D}$ is a nonsingular matrix, same with the proof process of Theorem 7 , one can know that the pinning consensus can be achieved. Due to the space limitation, the details about the proof are omitted here.

Next, the pinning scheme will be proposed analytically.

Recently, there are lots of reports about pinning scheme for multiagent networks. Like in [34], the results indicated that the network with undirected topology can achieve consensus by random pinning or selecting the high connections nodes. This conclusion was different from the result in [16], which was lower cost could be realized when the nodes with small degree were selected to be pinned. In [35], it indicated that the network can reach consensus by pinning the root of the spanning tree associated with its topology. Meanwhile, it was shown in $[16,18,36]$ that the nodes should be pinned if their out-degrees are bigger than in-degrees. In [21, 22, 24], it is shown that the root node (cannot be influenced by other nodes) should be pinned and the nodes satisfying the adaptive pinning scheme according to the degree-differences should also be selected. The results in [37] revealed that both the intra-act nodes with zero in-degree and the interact nodes should be simultaneously controlled to guarantee the realization of pinning consensus. In [38], the result indicated that the synchronization for the second-order multiagent network could be achieved when pinning one node with small coupling strength.

With respect to pinning control, there are two typical problems that need to be concerned; those are how to choose and how many nodes should be selected to be pinned, respectively. In application, it is not only costly but also impossible and unnecessary to directly control all the nodes in a network, especially when the node number of network is large. Therefore, inspired by the related research work mentioned above and based on the node connectivity of the system, we will propose our step-by-step comprehensive pinning scheme.

Step 1. The nodes with zero in-degree need to be pinned, which can ensure that the matrix $L+\widehat{D}$ is nonsingular. This key step will certainly guarantee the network to achieve consensus.

Step 2. The nodes can be given priority to be pinned if their out-degrees are bigger than in-degrees. This step can improve the convergence rate of the system, but it is worth noting that whether this type of node is selected or not cannot affect the convergence performance of the system.

Step 3. According to the specific situation, the other nodes of the network can be randomly pinned for the possibility of improving convergence rate of the system. This step may be determined by the trade-off mentioned above.

Remark 10. From the results in Theorems 5 and 7, it is not difficult to find that the random scheme cannot fully ensure the achievement of the pinning consensus. Meanwhile, most of the scheme listed above can be considered as a special case of our proposed strategy. In general, as we know, the more nodes are controlled, the faster the networks converge. But in fact, this rule does not always sound like that. It can be verified in the examples. Simultaneously, the trade-off between the control cost and the convergence rate of the system is also an important factor when introducing pinning control.

3.2. Adaptive Pinning Consensus for Discrete-Time Multiagent Network. Suppose a discrete-time multiagent network $g^{\prime}$ consists of $N$ agents, each of which described as follows:

$$
\begin{aligned}
x_{i}(t+1)= & x_{i}(t)+u_{i}(t), \quad i \in \mathbb{N}, \\
u_{i}(t)= & \varepsilon \sum_{j=1}^{N} G_{i j}\left(x_{j}(t)-x_{i}(t)\right) \\
& -\varepsilon d_{i}\left(x_{i}(t)-x^{*}\right), \quad i \in \mathbb{N},
\end{aligned}
$$

where $x_{i}(t), u_{i}(t) \in \mathbb{R}^{n}$ denote the state vector and the feedback controllers of the node $v_{i}$ at time $t$, respectively; $\varepsilon$ is the coupling strength of the system; $x^{*} \in \mathbb{R}^{n}$ is the desired equilibrium state. Control gains $d_{i}$ are defined as follows: $d_{i}>0$ if node $v_{i}$ is pinned; otherwise, $d_{i}=0$. Consider the protocol of adaptive control as follows:

$$
\dot{d}_{i}=\left(x_{i}(t)-x^{*}\right)^{T}\left(x_{i}(t)-x^{*}\right), \quad i \in \mathbb{N}
$$

From Definition 1, we know that it aims to design suitable controllers for network $g^{\prime}$ to satisfy

$$
\lim _{t \rightarrow+\infty}\left\|x_{i}(t)-x^{*}\right\|=0, \quad i \in \mathbb{N} .
$$


Theorem 11. Suppose that network (15) contains $N$ nodes and the coupling strength $\varepsilon>0$; the prospective pinning consensus for network (15) with general connected topology can be achieved asymptotically if $\varepsilon\left(d_{i}+2 l_{i i}\right) \leq 2$ holds, where $d_{i}$ are determined by the adaptive protocol (17) if node $v_{i}$ is pinned; otherwise, $d_{i}=0 . l_{i i}$ is the diagonal elements of the Laplacian matrix $L$.

Proof. Define the error system as

$$
e_{i}(t)=x_{i}(t)-x^{*}, \quad i \in \mathbb{N},
$$

and in view of (15) and (16), we can obtain the following error system:

$$
e_{i}(t+1)=e_{i}(t)+\varepsilon \sum_{j=1}^{N} a_{i j}\left(e_{j}(t)-e_{i}(t)\right)-\varepsilon d_{i} e_{i}(t),
$$

$$
i \in \mathbb{N} \text {. }
$$

Based on the definition of Laplacian matrix, (20) can be written as

$$
e_{i}(t+1)=e_{i}(t)-\varepsilon \sum_{j=1}^{N} l_{i j} e_{j}(t)-\varepsilon d_{i} e_{i}(t), \quad i \in \mathbb{N} .
$$

Let $e(t)=\left[e^{T}{ }_{1}(t), e^{T}{ }_{2}(t), \ldots, e^{T}{ }_{N}(t)\right]^{T}$; rewrite (7) in the matrix form as

$$
e(t+1)=\left[I_{N}-\varepsilon(L+D)\right] e(t),
$$

where $D=\operatorname{diag}\left\{d_{1}, d_{2}, \ldots, d_{N}\right\}, L$ is the Laplacian matrix, and $I_{N}$ is identity matrix. Suppose that $\lambda_{i}$ are the eigenvalues of matrix $I_{N}-\varepsilon(L+D), i=1,2, \ldots, N$; based on the theory of system stability, we can know that consensus of the network described by (22) could be achieved asymptotically when $\left|\lambda_{i}\right|<1, i=1,2, \ldots, N$ holds. By the Gershgorin disc theorem, we can obtain

$$
\left|\lambda_{i}-\left[1-\varepsilon\left(d_{i}+l_{i i}\right)\right]\right| \leq \varepsilon l_{i i}, \quad i=1,2, \ldots, N .
$$

In view of inequation (23), the following inequation can be obtained after some steps of calculation:

$$
\varepsilon\left(d_{i}+2 l_{i i}\right) \leq 2 .
$$

The proof of Theorem 11 is completed.

Remark 12. In Theorem 11, the algebraic criterion is obtained analytically, which is easy to be verified.

\section{Simulation Examples}

In this section, some simulation examples are illustrated to show the effectiveness of the results we proposed.

Example 13. In this simulation, we suppose the directed network system (3) with eight identical nodes and the topology is shown in Figure 1. Evidently, the topology of network (3) is not symmetrical and even owns no spanning directed tree. In

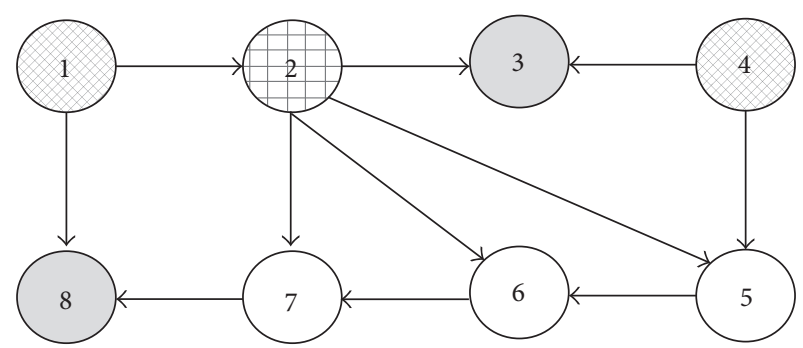

FIGURE 1: Directed interaction topology of the network.
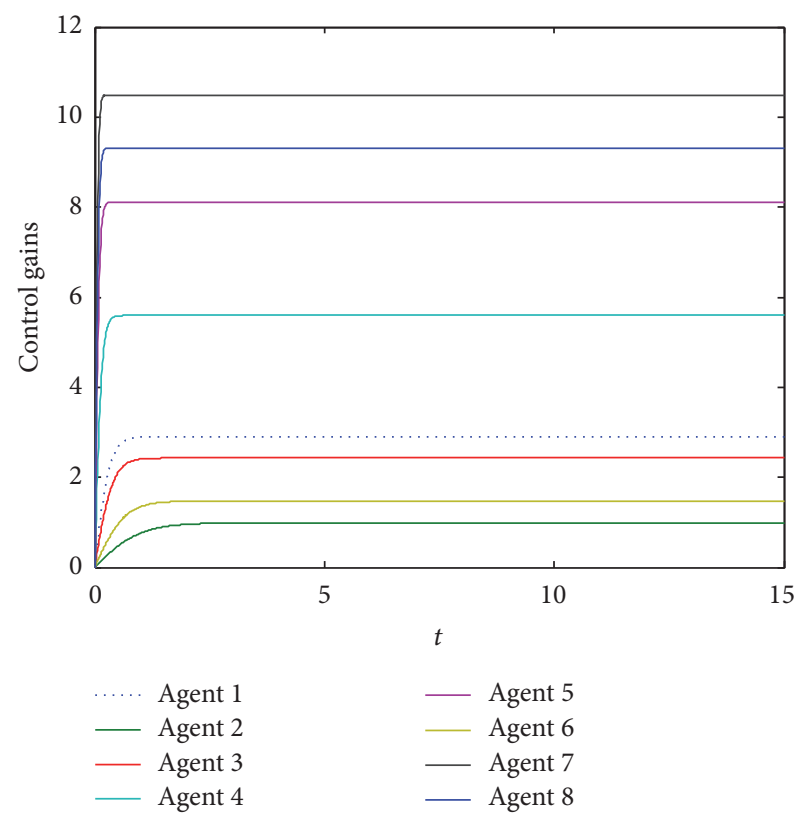

FIGURE 2: Time evolution of the adaptive control gains.

Figure $1, v_{1}$ and $v_{4}$ are the nodes with zero in-degree. $v_{3}$ and $v_{8}$ are the nodes whose in-degree is equal to their out-degree. Being different from the node $v_{2}$, the nodes $v_{5}, v_{6}$, and $v_{7}$ are the nodes whose in-degrees are bigger than their out-degrees.

For simplicity, we set $a_{i j}=1, i \neq j, c=1$, and $\Gamma=1$. Without loss of generality, we randomly generated the initial states of the agents from 0 to 20 . Meanwhile, we suppose the desired equilibrium state $x^{*}=6.0$. Namely, we aim to drive all the nodes in network (3) to reach the desired state 6.0.

By (9), it is easy to obtain the value of the control gains illustrated in Figure 2 . The details are $\widehat{d}_{1}=2.896, \widehat{d}_{2}=0.9884$, $\widehat{d}_{3}=2.428, \widehat{d}_{4}=5.594, \widehat{d}_{5}=8.113, \widehat{d}_{6}=1.474, \widehat{d}_{7}=10.49$, and $\widehat{d}_{8}=9.316$.

Through simple calculation, one can get the eigenvalues of $L+\widehat{D}$ being $11.3160,2.4900,3.4740,4.4280,10.1130,1.9884$, 2.8960 , and 5.5940, respectively. Meanwhile, it is also easy to verify that the matrix $L+\widehat{D}$ is an M-matrix. So the conditions in Theorems 5 and 7 and Corollary 9 are all satisfied.

Next, we will verify the validity of our theoretical findings from the following different cases, and the results are illustrated in Figure 3, respectively:

(i) Only node $v_{1}$ is pinned. 


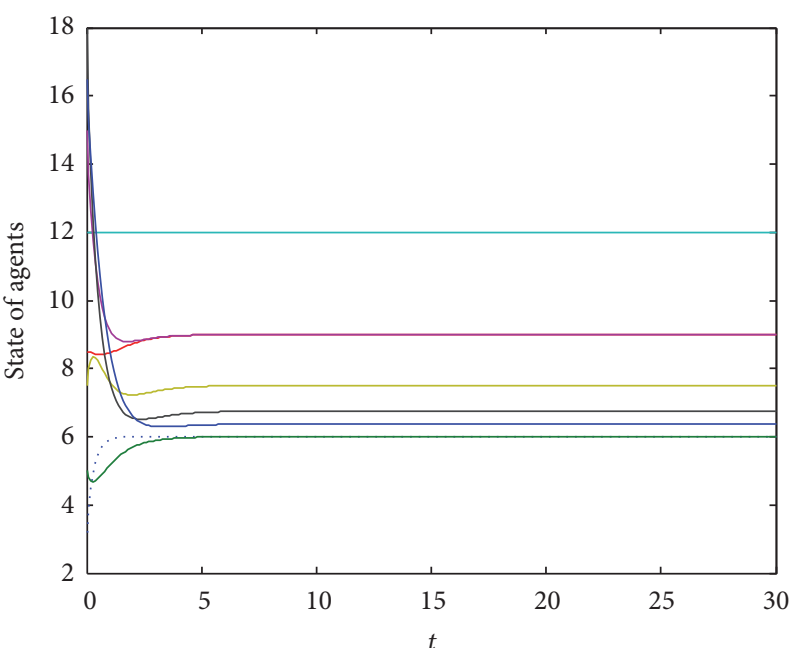

(a)

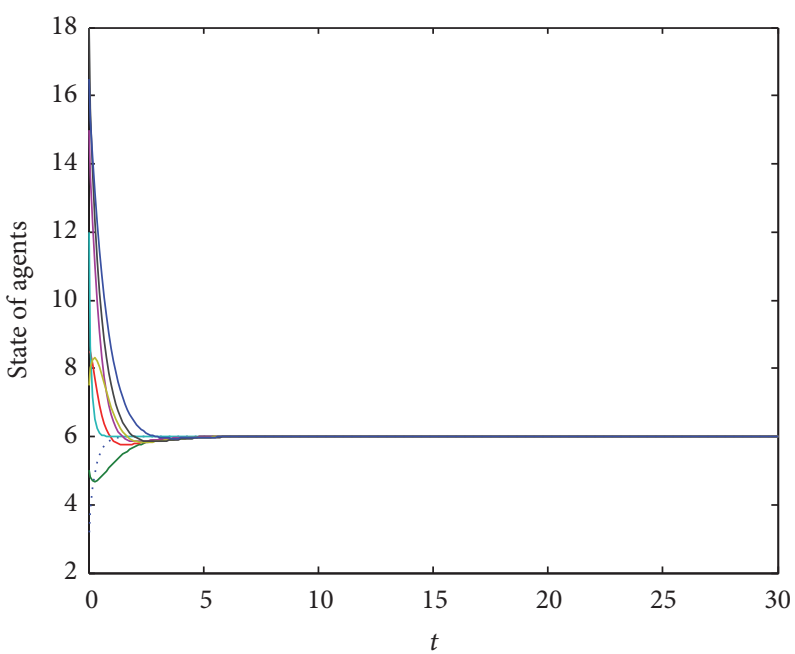

(c)

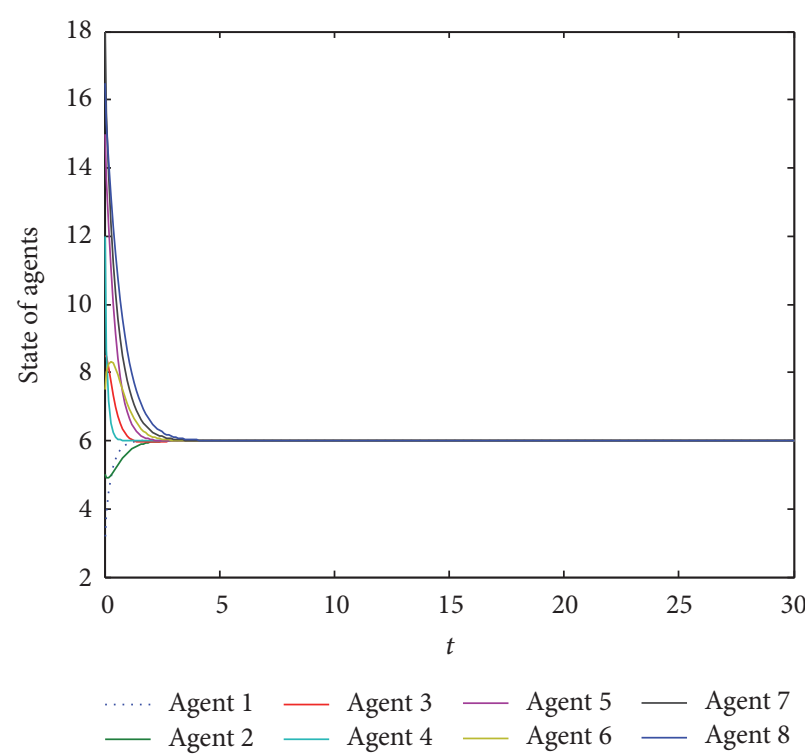

(e)

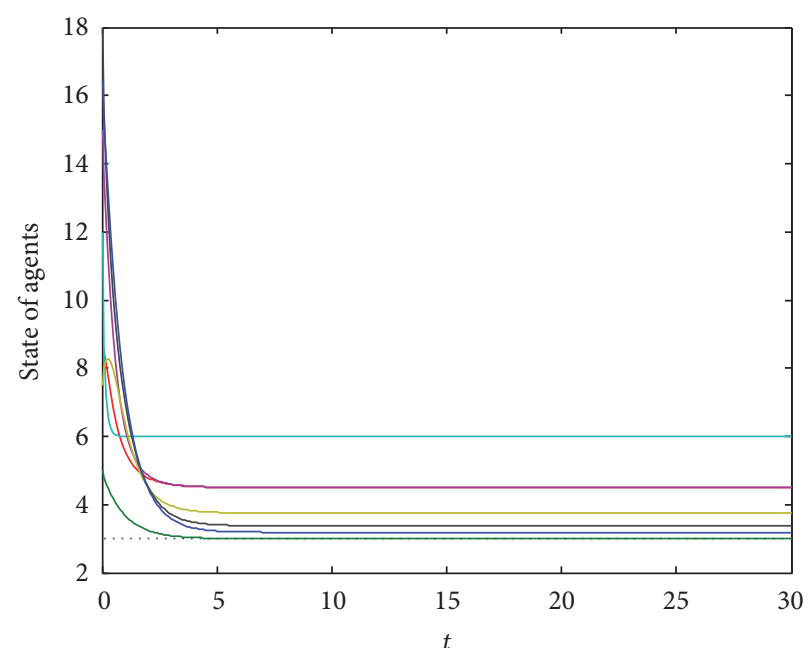

(b)

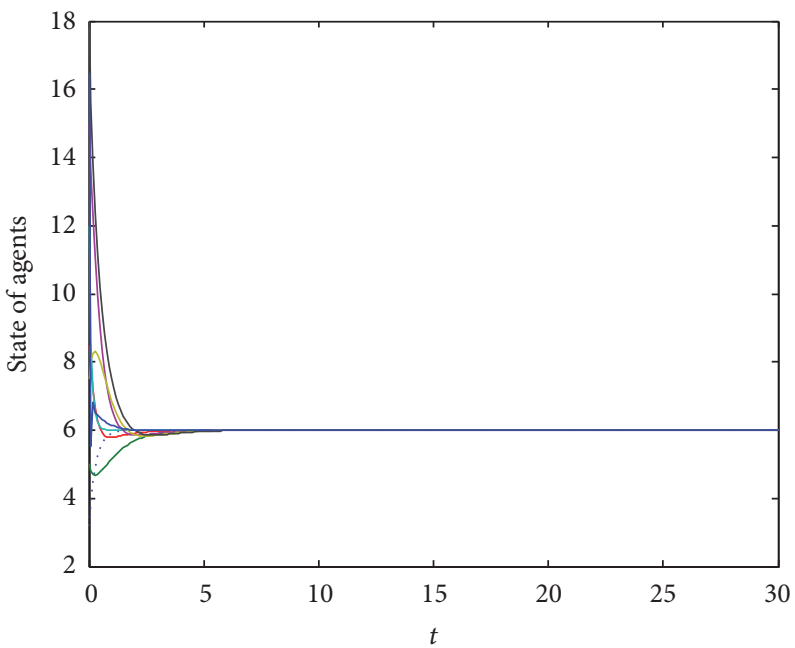

(d)

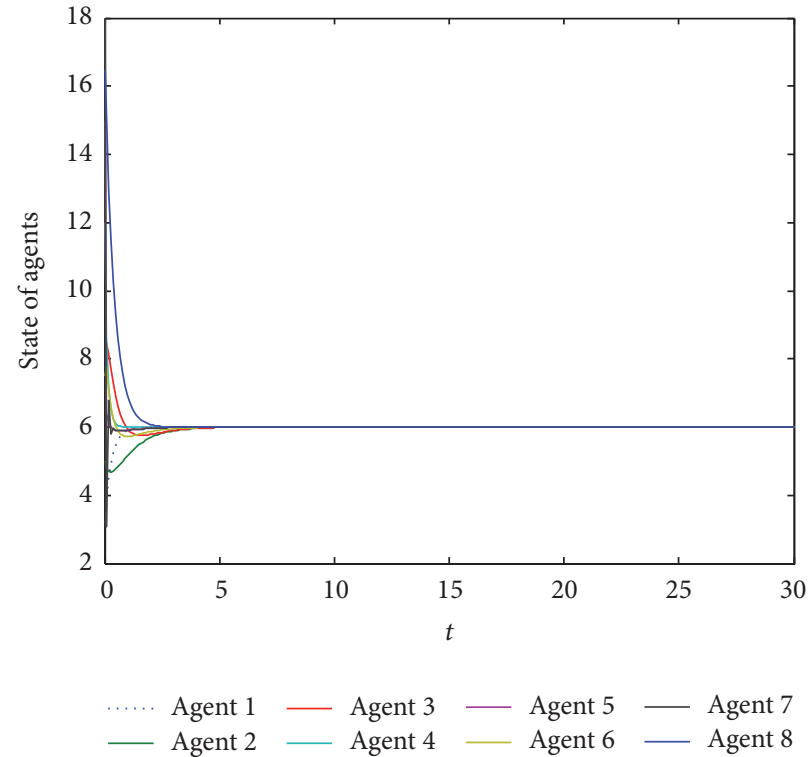

(f)

FIGURE 3: The state trajectories of the agents of network (3).(a) Only node $v_{1}$ is pinned. (b)Only node $v_{4}$ is pinned. (c) Nodes $v_{1}$ and $v_{4}$ are pinned. (d) Nodes $v_{1}, v_{3}, v_{4}$, and $v_{8}$ are pinned. (e) Nodes $v_{1}, v_{2}$, and $v_{4}$ are pinned. (f) Nodes $v_{1}, v_{4}, v_{5}, v_{6}$, and $v_{7}$ are pinned. 
TABLE 1: The relation between the selected nodes and the convergence time.

\begin{tabular}{|c|c|c|}
\hline ID & Selected nodes & $\begin{array}{c}\text { Convergence } \\
\text { time/computing step size }\end{array}$ \\
\hline 1 & 1,4 & 9.2 \\
\hline 2 & $1,2,4$ & 5.8 \\
\hline 3 & $1,3,4$ & 9.2 \\
\hline 4 & $1,2,3,4$ & 5.8 \\
\hline 5 & $1,8,4$ & 7 \\
\hline 6 & $1,2,8,4$ & 4.1 \\
\hline 7 & $1,3,8,4$ & 7 \\
\hline 8 & $1,2,3,8,4$ & 4.1 \\
\hline 9 & $1,5,4$ & 8.9 \\
\hline 10 & $1,2,5,4$ & 4 \\
\hline 11 & $1,6,4$ & 8.7 \\
\hline 12 & $1,2,6,4$ & 5.1 \\
\hline 13 & $1,7,4$ & 6.9 \\
\hline 14 & $1,2,7,4$ & 4.5 \\
\hline 15 & $1,5,6,4$ & 8.5 \\
\hline 16 & $1,2,5,6,4$ & 4.4 \\
\hline 17 & $1,5,7,4$ & 6.5 \\
\hline 18 & $1,2,5,7,4$ & 4.1 \\
\hline 19 & $1,6,7,4$ & 6.4 \\
\hline 20 & $1,2,6,7,4$ & 4.3 \\
\hline 21 & $1,5,6,7,4$ & 6.2 \\
\hline 22 & $1,2,5,6,7,4$ & 4.2 \\
\hline 23 & All nodes & 2.4 \\
\hline 24 & All except 2 & 4 \\
\hline
\end{tabular}

(ii) Only node $v_{4}$ is pinned.

(iii) Nodes $v_{1}$ and $v_{4}$ are pinned.

(iv) Nodes $v_{1}, v_{3}, v_{4}$, and $v_{8}$ are pinned.

(v) Nodes $v_{1}, v_{2}$, and $v_{4}$ are pinned.

(vi) Nodes $v_{1}, v_{4}, v_{5}, v_{6}$, and $v_{7}$ are pinned.

From the results shown in Figures 3(a), 3(b), and 3(c), it can be found that the pinning consensus cannot be achieved if the nodes with zero in-degree are not selected to be pinned, such as the nodes $v_{1}$ and $v_{4}$. Meanwhile, when the node $v_{2}$ whose out-degree is bigger than in-degree is added to be pinned, the convergence rate of the system can be improved significantly which can be seen from the comparison results shown in Figures 3(c)-3(f). In addition, we also find that the converge speed of the system will not be accelerated even if more nodes are pinned. That is to say, higher control costs do not mean that there will be a better convergence performance. So it reveals that the connectivity of the nodes plays key role in improving the convergence performance of the system. Next, a series of comparative experiments have been made, and the results are shown in Table 1 which can further verify the validity of the proposed pinned scheme which can provide certain references in the application.

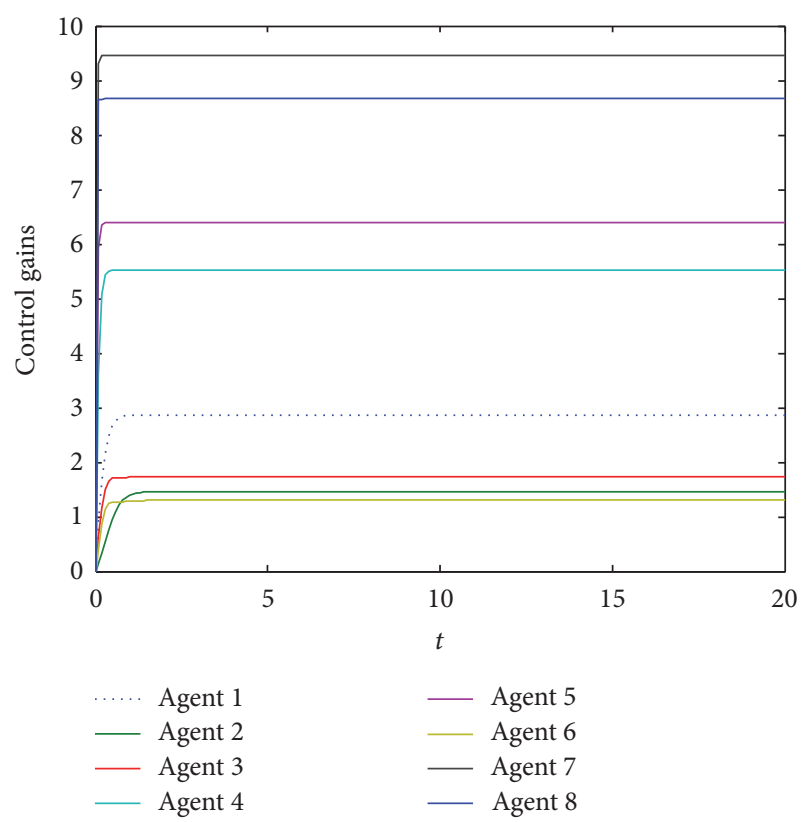

Figure 4: Time evolution of the adaptive control gains.

Example 14. In this simulation, it aims to verify the correctness of the results in Theorem 11. For simplicity, we also consider the dynamic networks (15) with the topology shown in Figure 1. Similarly, suppose the desired equilibrium state $x^{*}=6.0$, and the initial states of the agents in the network are generated randomly from 0 to 20 . By inequation (24), we can know that $\varepsilon<1 / 2$ holds. Without loss of generality, set $\varepsilon=0.1$. So the time evolution of the adaptive control gains is shown in Figure 4 by (17). Then we can get the diagonal matrix $D=\{2.867,1.461,1.735,5.526,6.404,1.302,9.465,8.675\}$. It is not difficult to verify that the algebraic conditions in Theorem 11 are all satisfied. The state trajectories of the agents of network (15) under different cases are shown in Figure 5. From the results, it is obviously known that the consensus can be realized when the zero in-degree nodes are selected to be pinned. Meanwhile, the convergence performance of the system can be obviously improved if the nodes whose out-degrees are bigger than in-degrees are also chosen to be pinned. In addition, like the results in Example 13, we also find out the fact that the more the nodes which are pinned are, the faster the system will converge is not always true.

Next, we will verify the validity of the bound of the algebraic criterion in Theorem 11. For simplicity, we only reset $d_{7}=18$ and keep the other parameters not changed. It is easy to know that inequation (24) cannot be satisfied, and the state trajectories of the agents in the network (15) are illustrated in Figure 6. Obviously, the pinning consensus of the system is not achieved. Therefore, the validity of the obtained algebraic criterion can be verified.

\section{Conclusion}

In this paper, pinning consensus problems for both continuous-time and discrete-time multiagent networks with general 


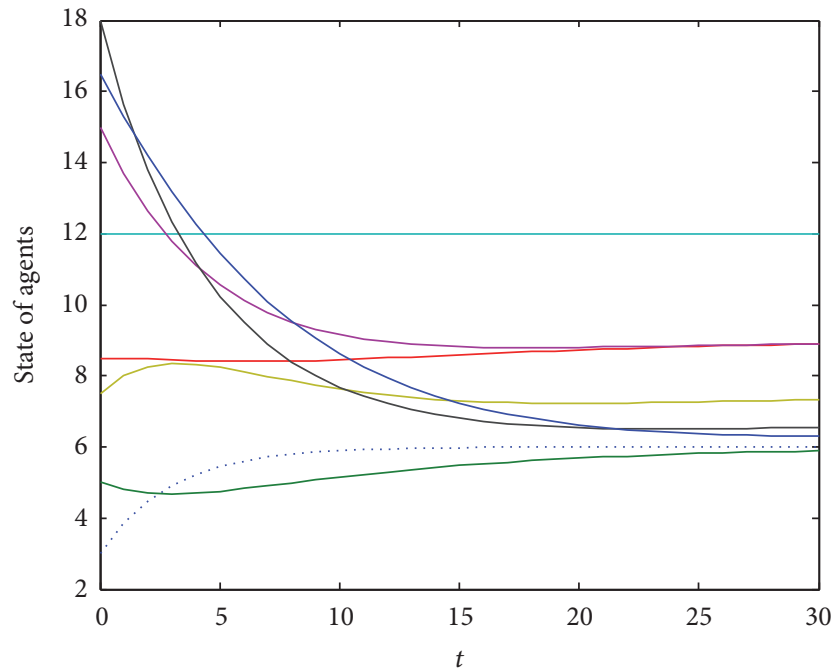

(a)

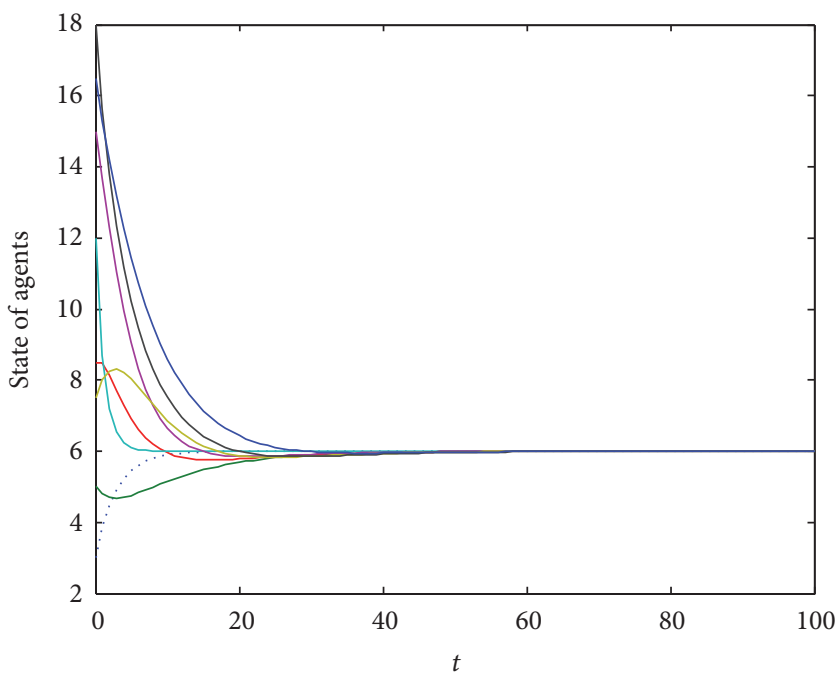

(c)

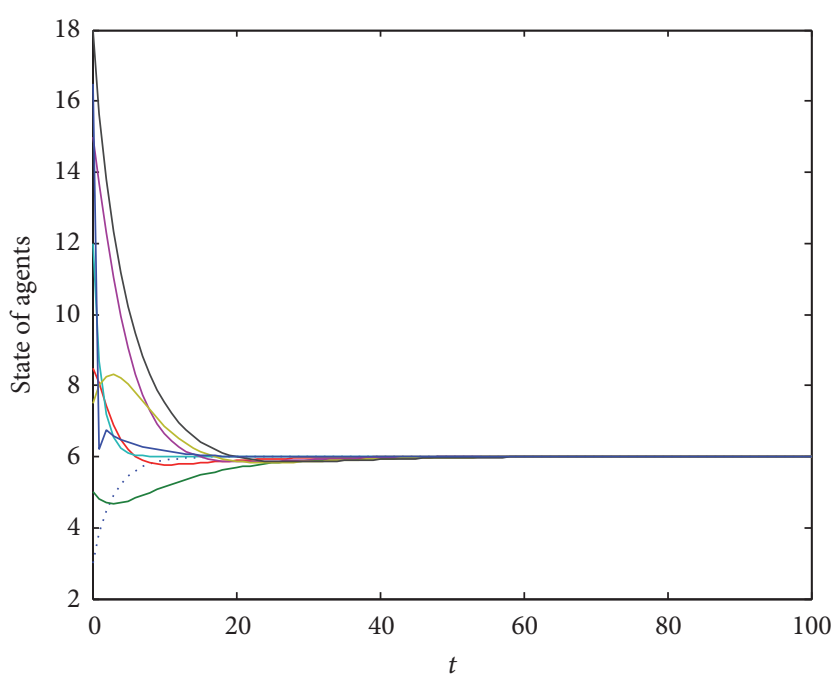

(e)

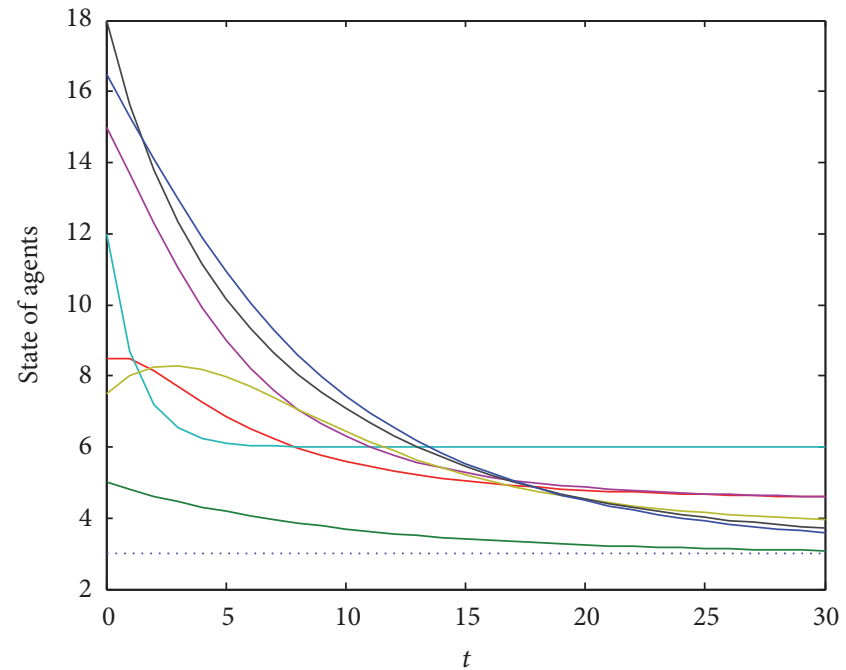

(b)

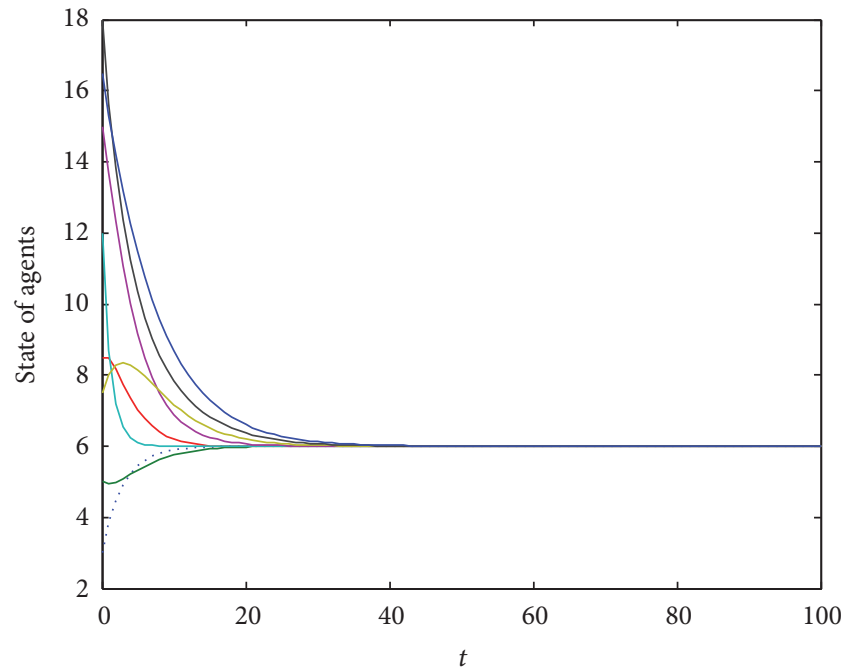

(d)

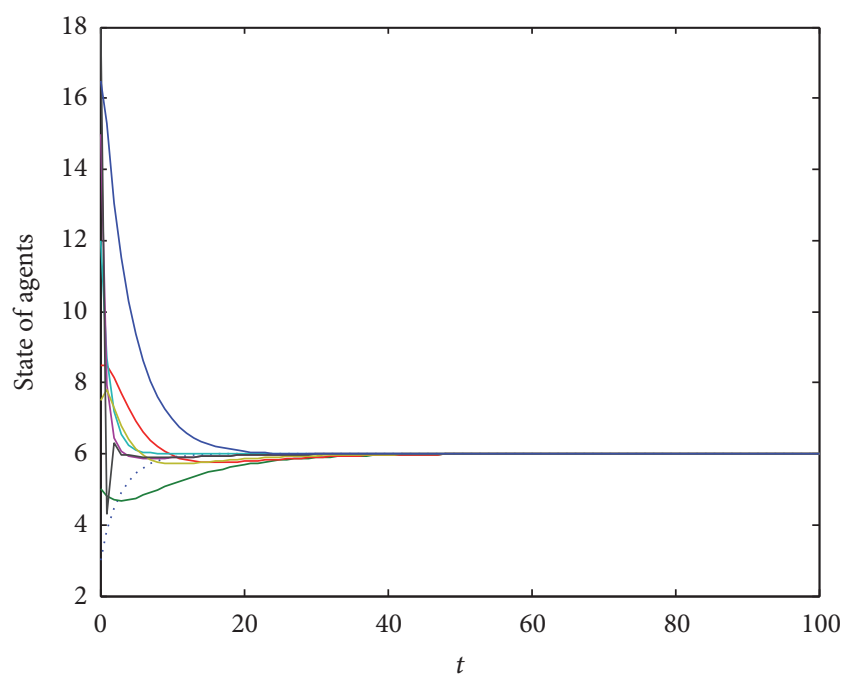

(f)

Figure 5: Continued. 


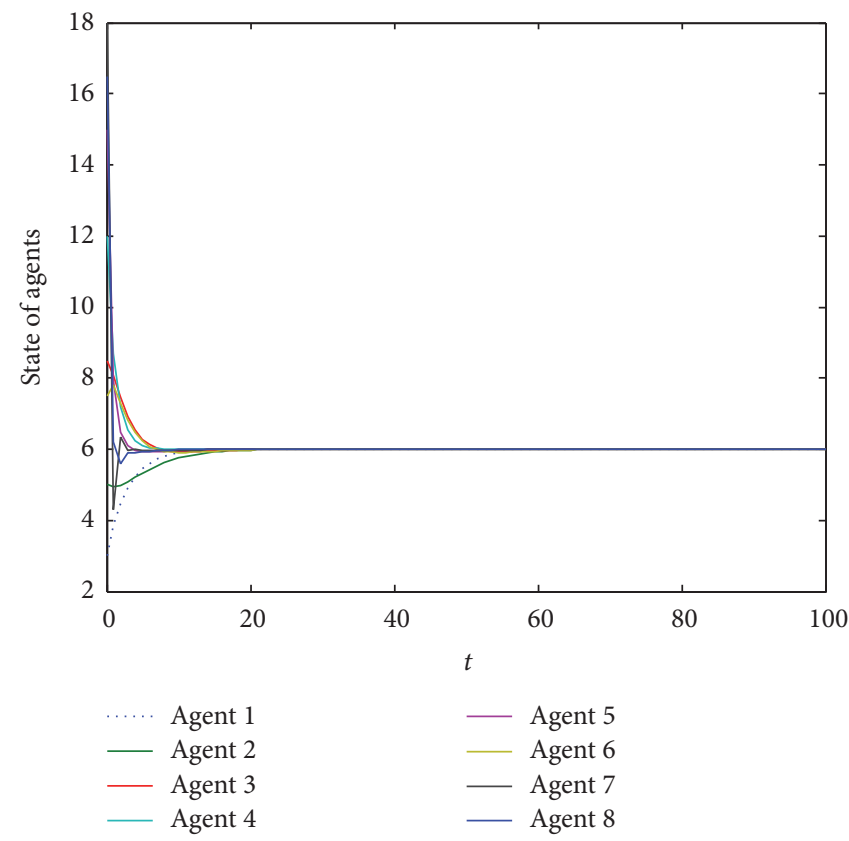

(g)

FIGURE 5: The state trajectories of the agents of network (15). (a) Only node 1 is pinned. (b) Only node 4 is pinned. (c) Nodes 1 and 4 are pinned. (d) Nodes 1, 2, and 4 are pinned. (e) Nodes 1, 3, 4, and 8 are pinned. (f) Nodes 1, 4, 5, 6, and 7 are pinned. (g) All nodes are pinned.

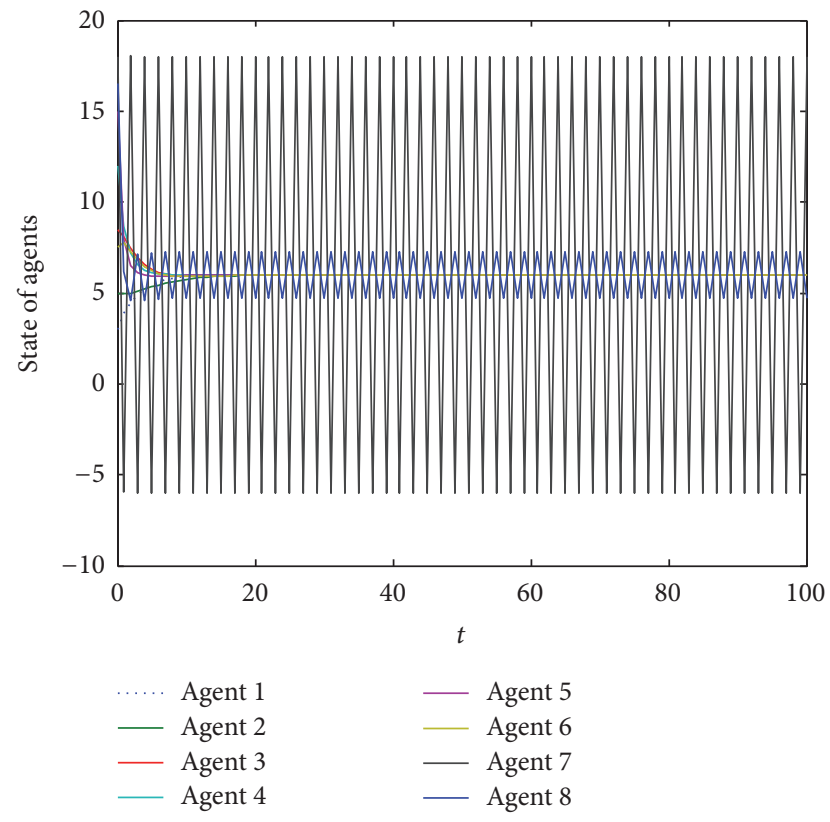

FIGURE 6: The state trajectories of the agents of network (15) when all nodes are pinned and $d_{7}=18$.

connected topology are elaborated, respectively. Different from the most existing research results, it is concluded that the topology of the system is not required to be symmetrical or contain a directed spanning tree. Some criteria are developed analytically, which can guarantee that the networks achieve pinning consensus by adopting adaptive control laws.
In addition, a comprehensive and effective pinning scheme is established which indicates that the nodes with zero indegree should be pinned for guaranteeing the convergence performance of the system, and then the nodes whose outdegrees are bigger than in-degrees can be preferred to other nodes when more nodes needed to be pinned to accelerate the convergence rate of the system. Meanwhile, it is also interesting to find that the conclusion may not always be true; that is, the convergence speed of the system will be accelerated with the increase of the pinned nodes. In order to improve the convergence rate of the system and reduce the control cost as well, the connectivity of the nodes is an important factor to decide whether or not a node should be pinned. Finally, the validity of all the obtained results is illustrated by several simulation examples.

\section{Competing Interests}

The authors declare that they have no competing interests.

\section{Acknowledgments}

This work was supported in part by the National Natural Science Foundation of China under Grant nos. 61472464, 61572091, and 61673080, in part by the Natural Science Foundation Project of Chongqing Science and Technology Commission under Grant no. cstc2014jcyjA40047, and in part by the Scientific and Technological Research Program of Chongqing Municipal Education Commission under Grant no. KJ1400403 and in part awarded by State Scholarship Fund of China Scholarship Council. 


\section{References}

[1] R. Olfati-Saber and R. M. Murray, "Consensus problems in networks of agents with switching topology and time-delays," IEEE Transactions on Automatic Control, vol. 49, no. 9, pp. 15201533, 2004.

[2] W. Ren and R. W. Beard, "Consensus seeking in multiagent systems under dynamically changing interaction topologies," IEEE Transactions on Automatic Control, vol. 50, no. 5, pp. 655661, 2005

[3] F. Xiao and L. Wang, "Asynchronous consensus in continuoustime multi-agent systems with switching topology and timevarying delays," IEEE Transactions on Automatic Control, vol. 53, no. 8, pp. 1804-1816, 2008.

[4] Y. Zhang and Y.-P. Tian, "Consentability and protocol design of multi-agent systems with stochastic switching topology," Automatica, vol. 45, no. 5, pp. 1195-1201, 2009.

[5] W. Yu, G. Chen, and M. Cao, "Some necessary and sufficient conditions for second-order consensus in multi-agent dynamical systems," Automatica, vol. 46, no. 6, pp. 1089-1095, 2010.

[6] P. Lin and Y. Jia, "Consensus of a class of second-order multiagent systems with time-delay and jointly-connected topologies," IEEE Transactions on Automatic Control, vol. 55, no. 3, pp. 778-784, 2010.

[7] W. Yu, W. X. Zheng, G. Chen, W. Ren, and J. Cao, "Second-order consensus in multi-agent dynamical systems with sampled position data," Automatica, vol. 47, no. 7, pp. 1496-1503, 2011.

[8] L. Ji, Q. Liu, and X. Liao, "On reaching group consensus for linearly coupled multi-agent networks," Information Sciences, vol. 287, pp. 1-12, 2014.

[9] H. Wang, X. Liao, T. Huang, and G. Chen, "Distributed parameter estimation in unreliable WSNs: quantized communication and asynchronous intermittent observation," Information Sciences, vol. 309, pp. 11-25, 2015.

[10] H. Li, X. Liao, T. Huang, W. Zhu, and Y. Liu, "Secondorder global consensus in multiagent networks with random directional link failure," IEEE Transactions on Neural Networks and Learning Systems, vol. 26, no. 3, pp. 565-575, 2015.

[11] T. Chen, X. Liu, and W. Lu, "Pinning complex networks by a single controller," IEEE Transactions on Circuits and Systems. I. Regular Papers, vol. 54, no. 6, pp. 1317-1326, 2007.

[12] W. Yu, G. Chen, and J. Lü, "On pinning synchronization of complex dynamical networks," Automatica, vol. 45, no. 2, pp. 429-435, 2009.

[13] J. Zhou, X. Wu, W. Yu, M. Small, and J. Lü, "Pinning synchronization of delayed neural networks," Chaos, vol. 18, no. 4, Article ID 043111, 2008.

[14] F. Chen, Z. Chen, L. Xiang, Z. Liu, and Z. Yuan, "Reaching a consensus via pinning control," Automatica, vol. 45, no. 5, pp. 1215-1220, 2009.

[15] X. Zhou, H. Feng, J. Feng, and Y. Zhao, "On synchronization of pinning-controlled networks with reducible and asymmetric coupling matrix," Communications and Network, vol. 3, no. 2, pp. 118-126, 2011.

[16] Q. Song and J. Cao, "On pinning synchronization of directed and undirected complex dynamical networks," IEEE Transactions on Circuits and Systems. I. Regular Papers, vol. 57, no. 3, pp. 672-680, 2010.

[17] M. Nariman, B. M. Mohammad, K. Jürgen, J. Lu, and A. Ahmad, "Pinning impulsive synchronization of complex dynamical networks," International Journal of Bifurcation and Chaos in
Applied Sciences and Engineering, vol. 22, no. 10, Article ID 1250239, 2012.

[18] Q. Song, F. Liu, J. Cao, and W. Yu, "Pinning-controllability analysis of complex networks: an M-matrix approach," IEEE Transactions on Circuits and Systems I: Regular Papers, vol. 59, no. 11, pp. 2692-2701, 2012.

[19] J. Qin and H. Gao, "A sufficient condition for convergence of sampled-data consensus for double-integrator dynamics with nonuniform and time-varying communication delays," IEEE Transactions on Automatic Control, vol. 57, no. 9, pp. 2417-2422, 2012.

[20] B. Liu, W. Lu, and T. Chen, "Pinning consensus in networks of multiagents via a single impulsive controller," IEEE Transactions on Neural Networks and Learning Systems, vol. 24, no. 7, pp. 11411149, 2013.

[21] Q. Song, F. Liu, J. Cao, and W. Yu, "M-matrix strategies for pinning-controlled leader-following consensus in multiagent systems with nonlinear dynamics," IEEE Transactions on Cybernetics, vol. 43, no. 6, pp. 1688-1697, 2013.

[22] H. Li, L. Xu, L. Xiao, and L. Lin, "Second-order leader-following consensus of nonlinear multi-agent systems via adaptive pinning control," in Proceedings of the 26th Chinese Control and Decision Conference (CCDC'14), pp. 586-591, Changsha, China, June 2014.

[23] W. Lu and F. M. Atay, "Local pinning of networks of multiagent systems with transmission and pinning delays," IEEE Transactions on Automatic Control, vol. 61, no. 9, pp. 2657-2662, 2015.

[24] H. Li, "Leader-following consensus of nonlinear multi-agent systems with mixed delays and uncertain parameters via adaptive pinning intermittent control," Nonlinear Analysis: Hybrid Systems, vol. 22, pp. 202-214, 2016.

[25] H.-X. Hu, Q. Xuan, W. Yu, C.-G. Zhang, and G. Xie, "Secondorder consensus for heterogeneous multi-agent systems in the cooperation-competition network: a hybrid adaptive and pinning control approach," Nonlinear Analysis: Hybrid Systems, vol. 20, pp. 21-36, 2016.

[26] J. Feng, J. Wang, C. Xu, and F. Austin, "Cluster synchronization of nonlinearly coupled complex networks via pinning control," Discrete Dynamics in Nature and Society, vol. 2011, Article ID 262349, 23 pages, 2011.

[27] T. Wang, T. Li, X. Yang, and S. Fei, "Cluster synchronization for delayed Lur'e dynamical networks based on pinning control," Neurocomputing, vol. 83, pp. 72-82, 2012.

[28] H. Su, Z. Rong, M. Z. Q. Chen, X. Wang, G. Chen, and H. Wang, "Decentralized adaptive pinning control for cluster synchronization of complex dynamical networks," IEEE Transactions on Cybernetics, vol. 43, no. 1, pp. 394-399, 2013.

[29] X. Liao and L. Ji, "On pinning group consensus for dynamical multi-agent networks with general connected topology," Neurocomputing, vol. 135, no. 5, pp. 262-267, 2014.

[30] Q. Ma, Z. Wang, and G. Miao, "Second-order group consensus for multi-agent systems via pinning leader-following approach," Journal of the Franklin Institute, vol. 351, no. 3, pp. 1288-1300, 2014.

[31] C. Xu, Y. Zheng, H. Su, M. Z. Chen, and C. Zhang, "Cluster consensus for second-order mobile multi-agent systems via distributed adaptive pinning control under directed topology," Nonlinear Dynamics, vol. 83, no. 4, pp. 1975-1985, 2016.

[32] Y. Cheng and H. Yu, "Adaptive group consensus of multi-agent networks via pinning control," International Journal of Pattern 
Recognition and Artificial Intelligence, vol. 30, no. 5, Article ID 1659014, 13 pages, 2016.

[33] A. Ronger and R. Charles, Topics in Matrix Analysis, Cambridge University Press, Cambridge, UK, 1991.

[34] X. F. Wang and G. Chen, "Pinning control of scale-free dynamical networks," Physica A, vol. 310, no. 3-4, pp. 521-531, 2002.

[35] C. Wu, "Localization of effective pinning control in complex networks of dynamical systems," in Proceedings of the IEEE International Symposium on Circuits and Systems, pp. 25302533, Seattle, Wash, USA, May 2008.

[36] Z. Yu, H. Jiang, C. Hu, and J. Yu, "Leader-following consensus of fractional-order multi-agent systems via adaptive pinning control," International Journal of Control, vol. 88, no. 9, pp. 17461756, 2015.

[37] Q. Ma and J. Lu, "Cluster synchronization for directed complex dynamical networks via pinning control," Neurocomputing, vol. 101, pp. 354-360, 2013.

[38] Y. Zhou, X. Yu, C. Sun, and W. Yu, "Robust synchronisation of second-order multi-agent system via pinning control," IET Control Theory \& Applications, vol. 9, no. 5, pp. 775-783, 2015. 


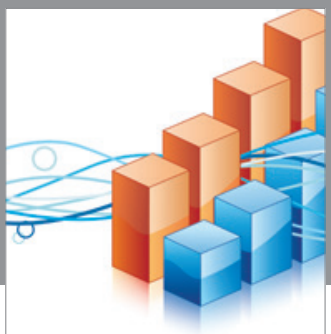

Advances in

Operations Research

vatem alat4

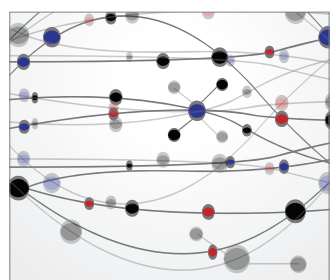

\section{The Scientific} World Journal
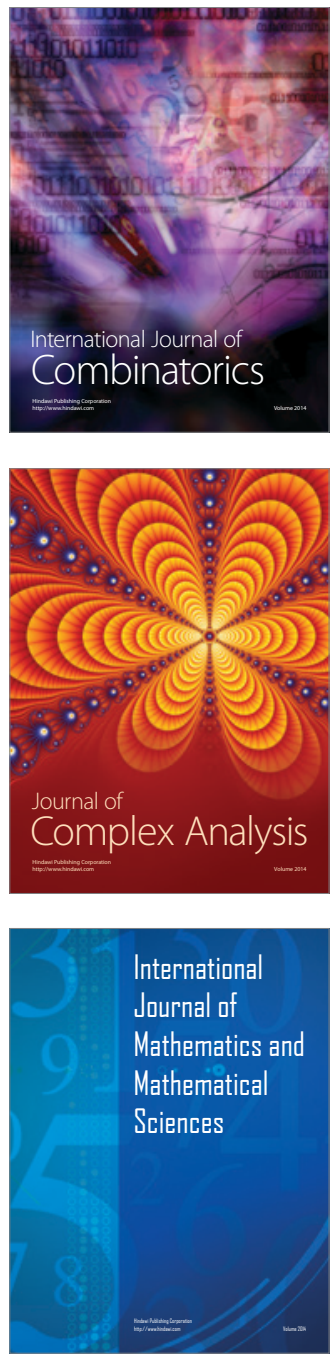
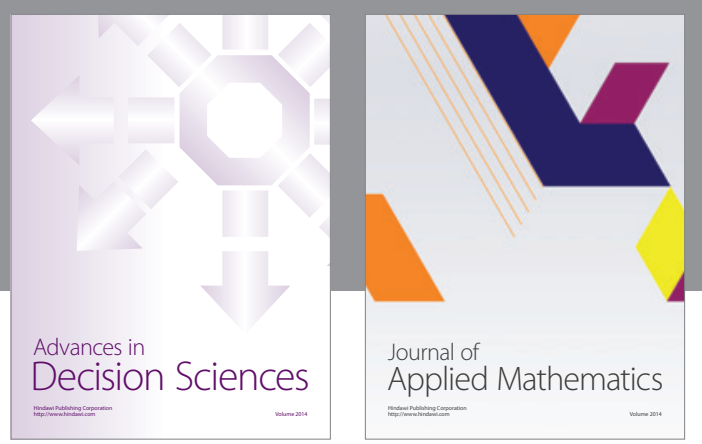

Algebra

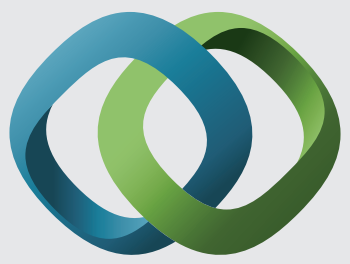

\section{Hindawi}

Submit your manuscripts at

http://www.hindawi.com
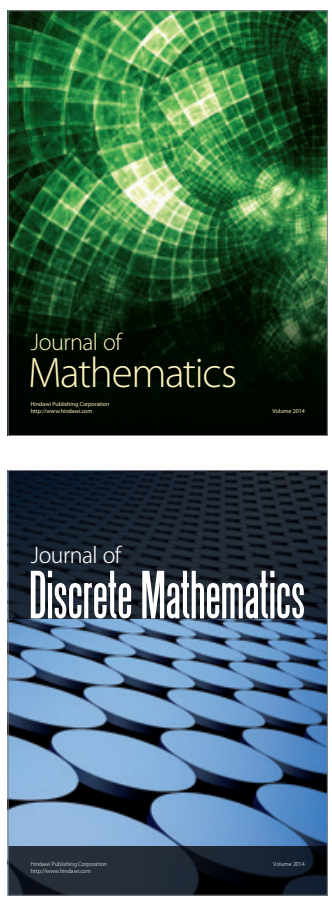

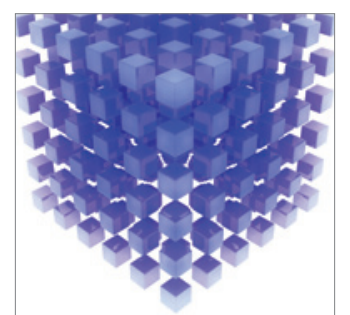

Mathematical Problems in Engineering
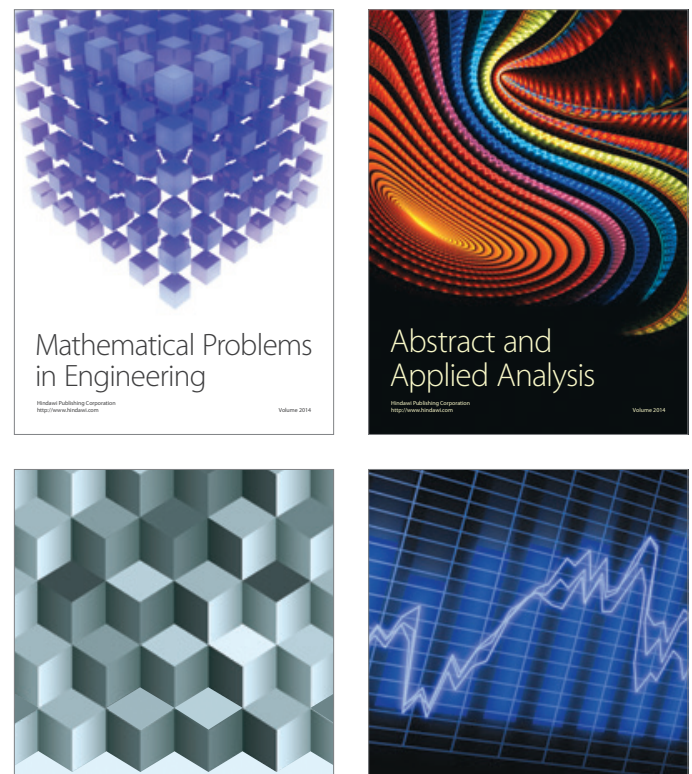

Journal of

Function Spaces

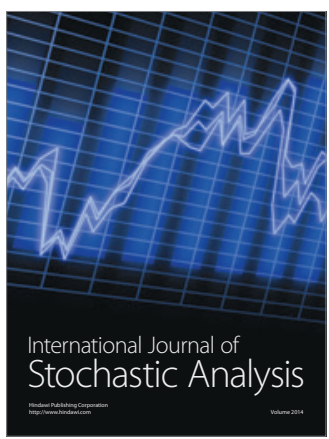

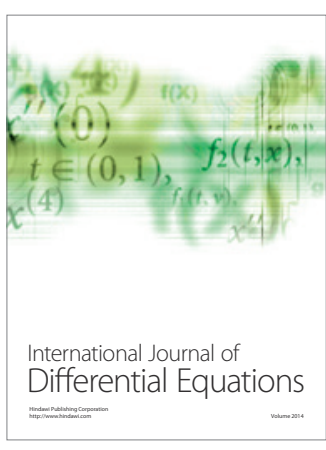
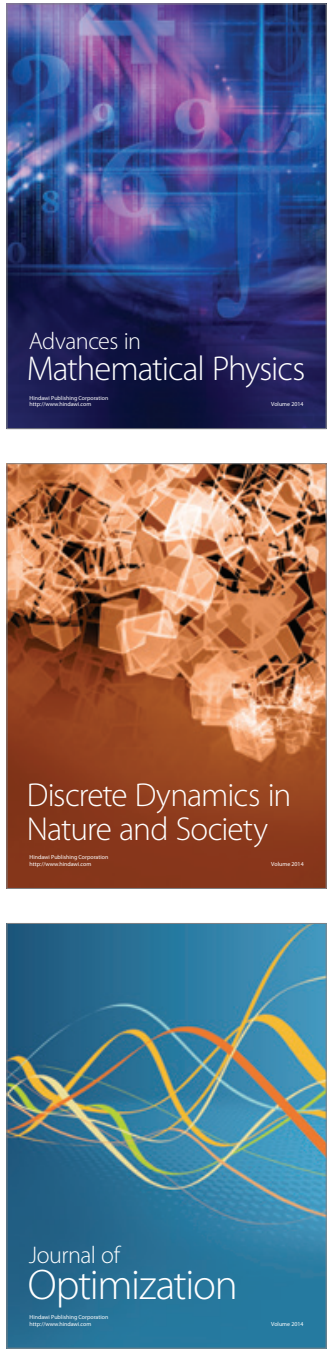